MONITORAMENTO AMBIENTAL

\title{
MÉTRICAS DE ECOLOGIA DE PAISAGEM EM AMBIENTE SIG PARA ANALISE DOS FRAGMENTOS FLORESTAIS DA BACIA DO RIO CLARO - PR
}

Guilherme Gobbi Teixeira - guilhermegob@gmail.com

Universidade Tecnológica Federal do Paraná - UTFPR, Câmpus Campo Mourão

Guilherme Redondo - guilherme.r.redondo@gmail.com

Universidade Tecnológica Federal do Paraná - UTFPR, Câmpus Campo Mourão

Heloise Beatriz Quesada - heloise_bq@ hotmail.com

Universidade Tecnológica Federal do Paraná - UTFPR, Câmpus Campo Mourão

Igor José Malfetoni Ferreira - igor_malfetoni@hotmail.com

Universidade Tecnológica Federal do Paraná - UTFPR, Câmpus Campo Mourão

Edivando Vitor do Couto - edivandocouto@ gmail.com

Universidade Tecnológica Federal do Paraná - UTFPR, Câmpus Campo Mourão

Resumo: A falta do planejamento para a utilização dos recursos naturais da paisagem pode causar danos irreversíveis, como a perda de biodiversidade. A dinâmica das paisagens pode ser compreendida por meio de métricas que mensuram e descrevem a estrutura espacial dos fragmentos florestais. Para propor a análise dos fragmentos florestais que compõem a subbacia hidrográfica do rio Claro foram utilizadas métricas de orla, forma e centro. Estas métricas foram calculadas através do SIG QGis ${ }^{\circledR}$ 2.10.1. Em relação a totalidade da sub-bacia, obteve-se o valor de 5,20\% de áreas florestais, em um total de 467 manchas. A área central das unidades, que sofre com menos intensidade dos efeitos adversos encontrados nas margens do fragmento, equivale a $14 \%$ da área total das manchas. De modo geral, a sub-bacia do rio Claro é intensamente fragmentada, com remanescentes florestais estreitos e isolados. $\mathrm{O}$ isolamento geográfico de fragmentos dificulta o contato entre espécies de diferentes áreas, comprometendo o fluxo gênico. Portanto, indica-se a implantação de corredores ecológicos para a movimentação e fluxo gênico de animais e dispersão de sementes.

Palavras-chave: Fragmentação, Métricas da paisagem, Biodiversidade, SIG. 


\section{INTRODUÇÃO E OBJETIVOS}

O grau de fragmentação da paisagem indica sua integridade ou qualidade ambiental, permitindo evidenciar tanto a quantidade de área desflorestada como também a estrutura do ambiente, por meio de indicadores como tamanho, forma e conectividade entre manchas florestais (IRGANG et al., 2007).

O primeiro efeito visível da fragmentação é a redução na riqueza das espécies, provocado por ações naturais, como o efeito de borda. As espécies instaladas nas regiões de borda são afetadas de forma negativa pelas interações com outras espécies que vivem na matriz. Outro efeito adverso seria o isolamento das populações inseridas no fragmento, sendo por vezes impossível o deslocamento entre unidades (RICKLEFS, 2010).

Uma alternativa que minimiza os efeitos da fragmentação são os corredores ecológicos, descritos como faixas estreitas de habitat que promovem movimento dos organismos de um fragmento para outro. Os corredores possuem também função de transporte de água, energia e minerais, de elemento barreira à passagem de espécies ou materiais e como recurso (BATISTA, 2014).

A dinâmica da paisagem pode ser interpretada através de métricas, que medem e descrevem a estrutura espacial dos fragmentos, classes de fragmentos ou da totalidade (BATISTA, 2014).

O Sistema de Informações Geográficas (SIG) tem sido uma importante ferramenta para os profissionais das ciências ambientais, pois é utilizado para visualizar e quantificar características da paisagem, procurando por padrões de como os organismos são afetados pela fragmentação (RICKLEFS, 2010).

O trabalho tem como objetivo efetuar uma análise métrica dos fragmentos que compõe a paisagem da sub-bacia hidrográfica do rio Claro, utilizando SIG afim de avaliar o estado atual dos fragmentos florestais.

\section{METODOLOGIA}

A sub-bacia do rio Claro encontra-se na bacia hidrográfica do rio Ivaí, localizada em sua totalidade no estado do Paraná, firmada no Segundo e Terceiro Planalto Paranaenses (LELI et al., 2010). O clima da bacia hidrográfica é, segundo a classificação de Köppen, Cfa, subtropical, com verões quentes, geadas pouco frequentes e concentração das chuvas nos meses de verão, sem estação seca definida, e $\mathrm{Cfb}$, temperado, com verões frescos e sem estação seca definida (IAPAR, s/d). Os solos abrangentes na bacia são os Latossolos, Argissolos, Neossolos Flúvicos e Nitossolos (ITCG, 2008). De acordo com o Manual Técnico da Vegetação Brasileira (2012) a cobertura vegetal remanescente é formada pela Floresta Ombrófila Mista, Floresta Estacional Semidecidual, savana e estepe.

Tendo em vista que a sub-bacia possui cerca de $2639,02 \mathrm{~km}^{2}$ de extensão, os municípios compreendidos e suas respectivas áreas $\left(\mathrm{km}^{2}\right)$ são: Engenheiro Beltrão (366.48), Marialva (303.59), Terra Boa (268.34), Maringá (210.43), Ourizona (177.01), Paiçandu (172.13), Floresta (157.75), Peabiru (150.70), Jussara (130.56), Doutor Camargo (117.76), São Jorge do Ivaí (107.42), Ivatuba (97.09), Araruna (88.92), Mandaguaçu (71.93), Itambé (70.95), Sarandi (58.85), Campo Mourão (49.70) e Presidente Castelo Branco (39.41). 
A Figura 1 ilustra a localização da sub-bacia em relação a bacia hidrográfica do

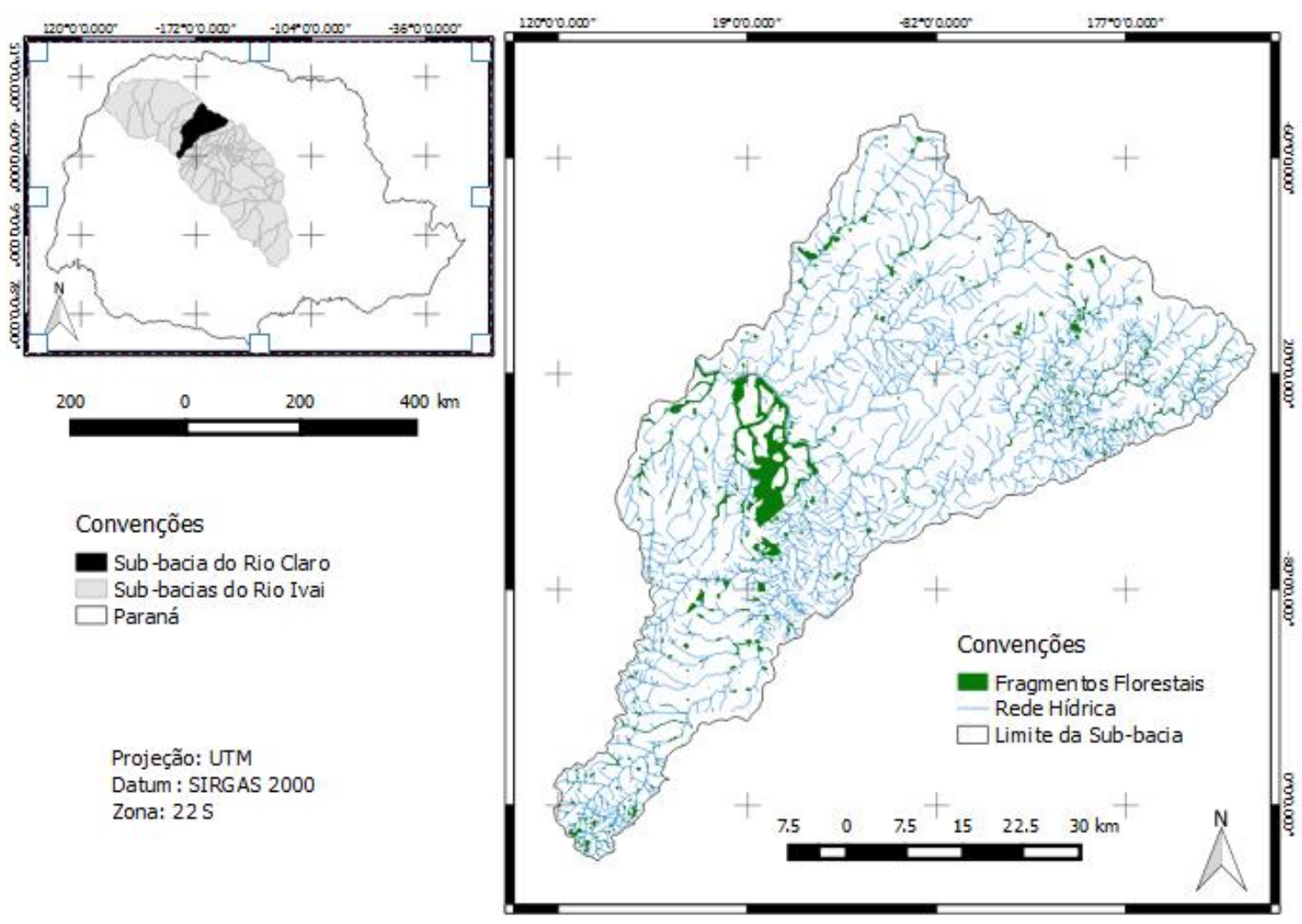

Figura 1 - Localização da sub-bacia do rio Claro.

Em um banco de dados disponibilizado pelo Projeto Rede Hídrica, desenvolvido pelo Instituto de Águas Paraná e patrocinado pela Copel, foi definida para o estudo a subbacia do rio Claro, dentre todas que compõem a bacia hidrográfica do rio Ivaí, tendo o SIG Qgis 2.10.1 como ferramenta computacional. Com o banco de dados Fragmentos Florestais, disponibilizado pelo projeto SOS Mata Atlântica, foi possível visualizar os fragmentos contidos na sub-bacia e, através do Projeto Rede Hídrica, foram obtidas as informações referentes a rede hidrográfica. As características físicas do local foram analisadas a partir do recorte das camadas de hidrografia e fragmentos florestais da sub-bacia em questão.

As métricas da paisagem foram calculadas com a calculadora de campo do Qgis 2.10.1., juntamente com complemento Landscape Ecology Statistics (LecoS), que estão descritas na Tabela 2. 
Tabela 2 - Métricas da paisagem.

\begin{tabular}{|c|c|c|}
\hline Métricas & Parâmetros & Referências \\
\hline $\begin{array}{l}\text { Tamanho da mancha: } \\
\text { PS ou } A R E A=a_{i j}\end{array}$ & $\begin{array}{l}\mathrm{a}_{\mathrm{ij}}=\text { área }\left(\mathrm{m}^{2}\right) \quad \mathrm{da} \\
\text { mancha }\end{array}$ & $\begin{array}{l}\text { McGarial \& Marks, 1995; Leitão et } \\
\text { al., 2006; Hoechestetter, } 2009\end{array}$ \\
\hline $\begin{array}{l}\text { Tamanho médio das } \\
\text { manchas: } \\
\qquad M P S=\frac{\sum_{j=1}^{n} a_{i j}}{P N_{i}}\end{array}$ & $\begin{array}{lll}a_{i j}=\text { área }\left(\mathrm{m}^{2}\right) & \mathrm{da} \\
\text { mancha } & & \\
P N_{i}=\quad \text { número } & \mathrm{de} \\
\text { manchas da classe } & \end{array}$ & $\begin{array}{l}\text { McGarigal \& Marks, 1995; Wu et al., } \\
\text { 2003; Rempel et al., } 2012\end{array}$ \\
\hline $\begin{array}{l}\text { Índice de maior mancha: } \\
L P I=\frac{\max a_{i j}}{A R E A} * 100\end{array}$ & $\begin{array}{l}a_{i j}=\text { área }\left(\mathrm{m}^{2}\right) \mathrm{da} \\
\text { mancha } \\
\begin{array}{l}\mathrm{AREA}=\text { área total da } \\
\text { paisagem }\end{array}\end{array}$ & $\begin{array}{l}\text { McGarigal \& Marks, 1995; Wu et al., } \\
\text { 2002; Leitão et al., 2006; } \\
\text { Hoechestetter, } 2009\end{array}$ \\
\hline $\begin{array}{l}\text { Índice da menor mancha: } \\
S P I=\frac{\min a_{i j}}{A R E A} * 100\end{array}$ & $\begin{array}{l}a_{i j}=\text { área }\left(\mathrm{m}^{2}\right) \mathrm{da} \\
\text { mancha } \\
\begin{array}{l}\mathrm{AREA}=\text { área total da } \\
\text { paisagem }\end{array}\end{array}$ & Batista, 2014 \\
\hline $\begin{array}{l}\text { Razão perímetro/área: } \\
\qquad P A R A=\frac{P E R I M}{A R E A}\end{array}$ & $\begin{array}{l}\text { PERIM = perímetro da } \\
\text { mancha } \\
\begin{array}{l}\text { AREA }=\text { área da } \\
\text { mancha }\end{array}\end{array}$ & $\begin{array}{l}\text { Hoechestetter, 2009; McGarigal et } \\
\text { al., } 2012\end{array}$ \\
\hline $\begin{array}{l}\text { Tamanho da malha efetiva: } \\
M E S H=\frac{A R E A M^{2}}{A R E A}\end{array}$ & $\begin{array}{l}\text { AREAM = soma das } \\
\text { áreas das manchas } \\
\text { AREA = área total da } \\
\text { paisagem }\end{array}$ & $\begin{array}{l}\text { Jaeger, } 2000 \text { cit ind Hoechestetter, } \\
\text { 2009; McGarigal et al., } 2012\end{array}$ \\
\hline $\begin{array}{l}\text { Número de manchas: } \\
\qquad N P=n_{i}\end{array}$ & $\begin{array}{l}n_{i} \quad=\text { número de } \\
\text { manchas }\end{array}$ & $\begin{array}{l}\text { McGarigal \& Marks, 1995; Wu et al., } \\
\text { 2002; Leitão et al., 2006; Rempel et } \\
\text { al., 2012; McGarigal et al., } 2012\end{array}$ \\
\hline $\begin{array}{l}\text { Índice de Patton: } \\
C=\frac{P}{\sqrt{\pi A}}\end{array}$ & $\begin{array}{l}\mathrm{P}=\text { perímetro do } \\
\text { fragmento } \\
\mathrm{A}=\text { área do fragmento }\end{array}$ & Peterka, 2008 \\
\hline $\begin{array}{l}\text { Área do núcleo: } \\
\qquad C O R E=a_{i j}^{c} * \frac{1}{10000}\end{array}$ & $\begin{array}{l}a_{i j}^{c}=\text { área }\left(\mathrm{m}^{2}\right) \text { do } \\
\text { núcleo da mancha }\end{array}$ & http://plugins.qgis.org/plugins/LecoS/ \\
\hline $\begin{array}{l}\text { Densidade da mancha: } \\
P D=\frac{n_{i}}{A} *\left(10^{\wedge} 4\right) *(100)\end{array}$ & $\begin{array}{l}n_{i}=\text { número de } \\
\text { manchas na paisagem } \\
A=\text { área total da } \\
\text { paisagem }\left(\mathrm{m}^{2}\right)\end{array}$ & $\begin{array}{l}\text { McGarigal \& Marks, 1995; Wu et al., } \\
\text { 2002; Hoechestetter, } \\
\text { McGarigal et al., } 2012\end{array}$ \\
\hline
\end{tabular}

\section{RESULTADOS E DISCUSSÕES}

O número de fragmentos florestais da sub-bacia foi contabilizado em 467 unidades, tendo $0,29 \mathrm{~km}^{2}$ como área média dos fragmentos. É possível visualizar na Figura 2 a distribuição da área de todos os fragmentos florestais encontrados na região estudada. 

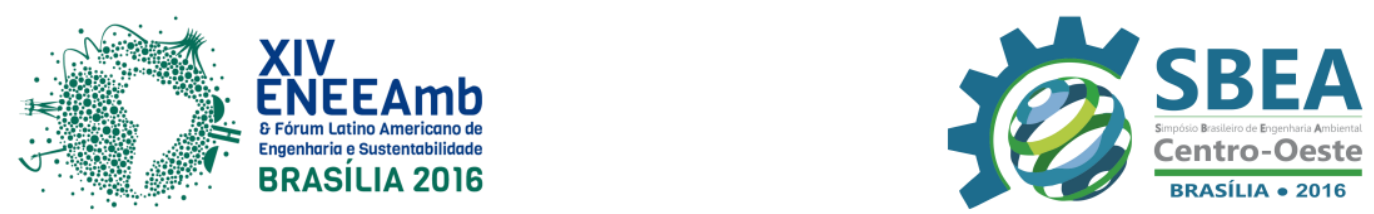

Observa-se que $75 \%$ dos fragmentos de área são inferiores a $0,2 \mathrm{~km}^{2}$ e $50 \%$ inferiores a 0,08 $\mathrm{km}^{2}$. O menor fragmento possui $0,00063 \mathrm{~km}^{2}$ de extensão e o maior, $48,55 \mathrm{~km}^{2}$, sendo esse o fragmento que mais se distancia da média e, portanto, o outlier que mais exerce influência sobre a distribuição.

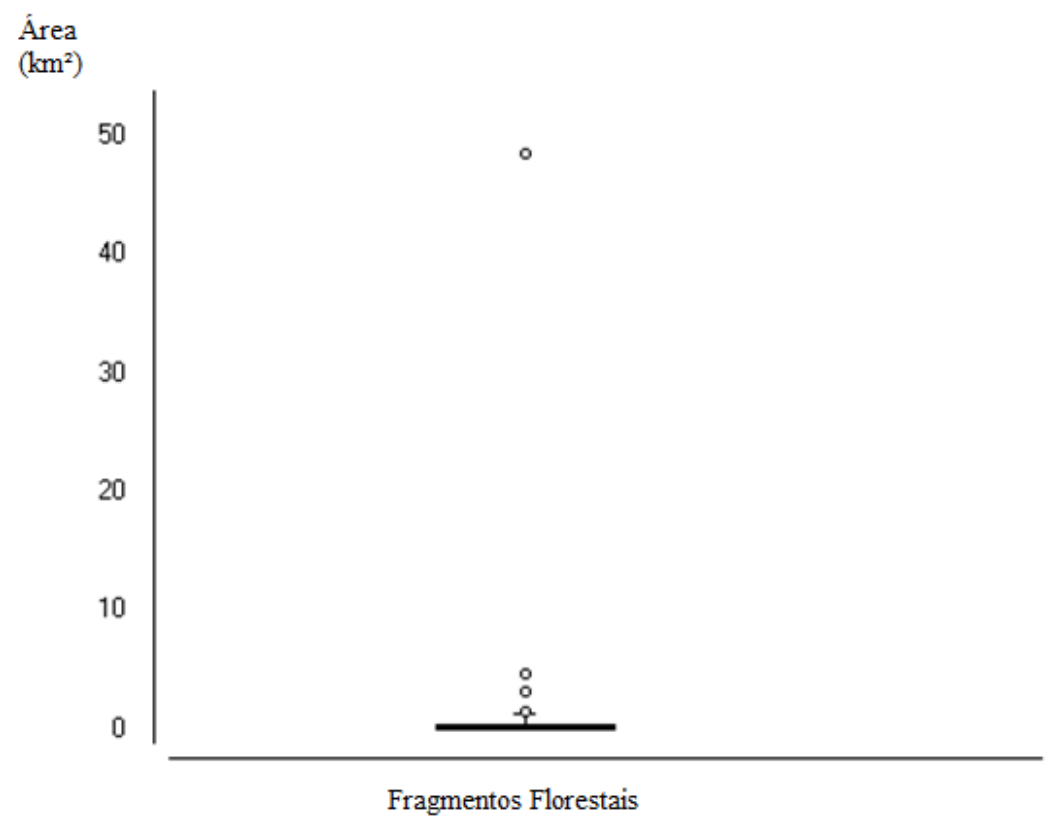

Figura 2 - Área dos fragmentos florestais da Bacia Hidrográfica do Rio Claro, PR.

A área total de manchas florestais foi equivalente a $137,32 \mathrm{~km}^{2}$, correspondendo a $5,20 \%$ da área total da sub-bacia. O índice da menor mancha foi de 0,000024\% em relação à paisagem, enquanto a maior mancha representa $1,83 \%$ desse índice. Percebe-se, pela porcentagem baixa dos índices, que as áreas menores não são significativas quando comparadas à matriz, e que mesmo os maiores fragmentos, quando relacionados a áreas maiores, não demonstram importância expressiva.

Quanto à forma, a relação perímetro-área média foi 0,016. O menor valor encontrado foi 0,0024 , já o maior, 0,25. Essa relação fornece informações sobre a circularidade do fragmento. A circularidade reage positivamente em relação ao efeito de borda, preservando com mais eficiência o núcleo da parcela, pois a influência externa nesse ambiente é amenizada pelo contorno da vegetação (IRGANG et al., 2007).

A menor relação perímetro-área encontrada refere-se ao maior fragmento, ilustrado pela Figura 3, já que sua forma se distancia de um círculo. Assim, mesmo possuindo a maior área, a unidade assume um efeito negativo para a conservação do meio, o que resulta em uma menor área de núcleo e, consequentemente, maiores efeitos de borda. Em contrapartida, o menor fragmento possui a maior relação perímetro-área (IRGANG et al., 2007).

A área núcleo dos fragmentos florestais equivale a $19,8 \mathrm{~km}^{2}$. Desse modo, observa-se que apenas $14 \%$ da área total das manchas sofrem com menos intensidade os efeitos adversos encontrados na margem do fragmento. 


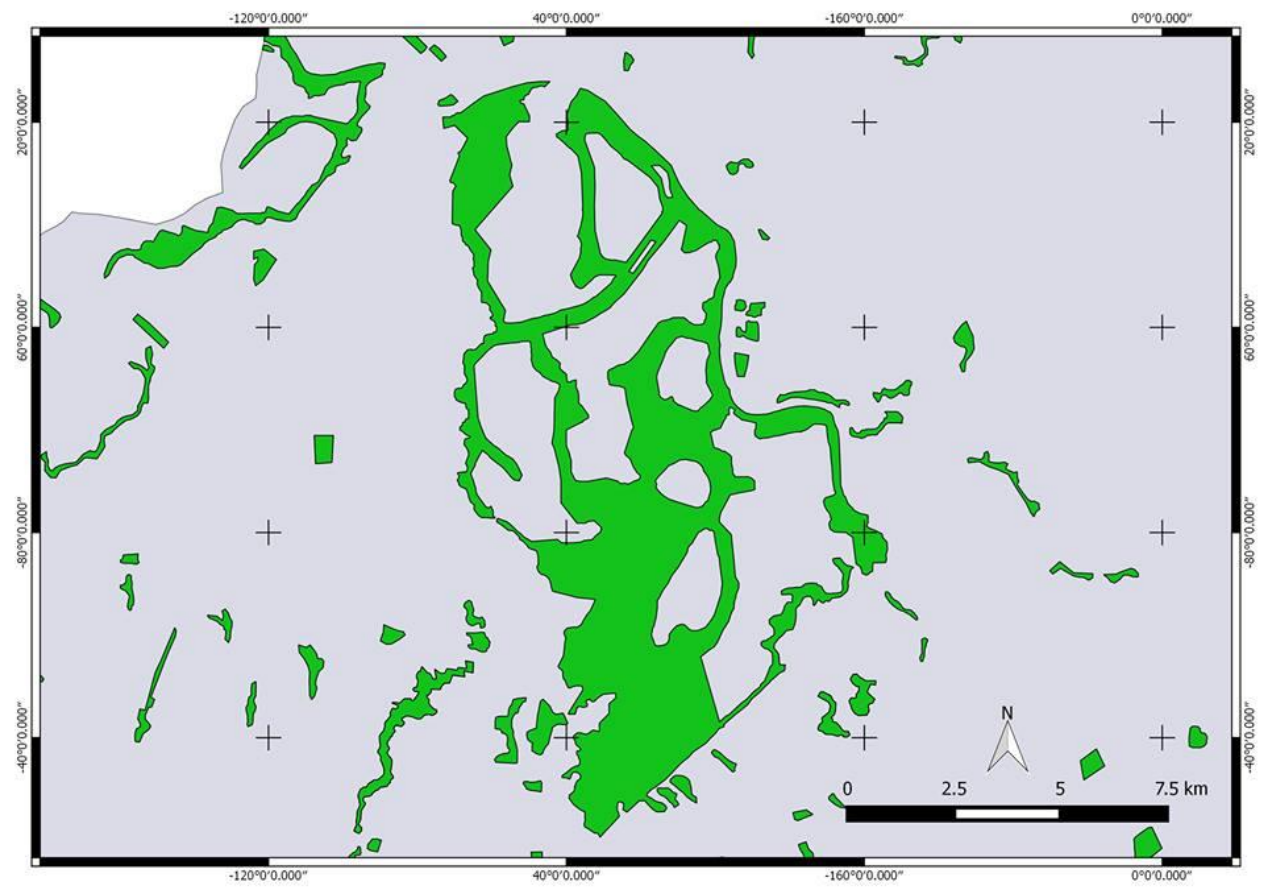

Figura 3 - Maior fragmento florestal da sub-bacia do rio Claro.

A Figura 4 representa a distribuição do índice de Patton dos fragmentos da subbacia do rio Claro. Em relação ao grau de complexidade de forma, os fragmentos possuem como média 3,19, sendo 2,11 o menor valor e 10,29 o maior. Notou-se ainda que a bacia possui $50 \%$ de seus fragmentos com índice de Patton inferior a 2,42 e 75\% inferior a 3,45. Tais índices se mostraram melhores do que aqueles encontrados em estudos similares sobre o efeito da fragmentação na bacia no rio Alonzo, Paraná, realizado por Ferreira et al. (2016). Para a bacia do rio Claro foram encontrados índices de Patton menores que os apresentados para a bacia do rio Alonzo, representando fragmentos com menor grau de complexidade de forma.

No entanto, os mesmos fragmentos apresentam áreas muito menores, o que para este caso, segundo Ferreira et al. (2016), pode influenciar diretamente em sua funcionalidade ecológica, sendo responsável por interagir de forma negativa com outros fatores como a área de núcleo e complexidade de forma e produzir maior efeito borda. 


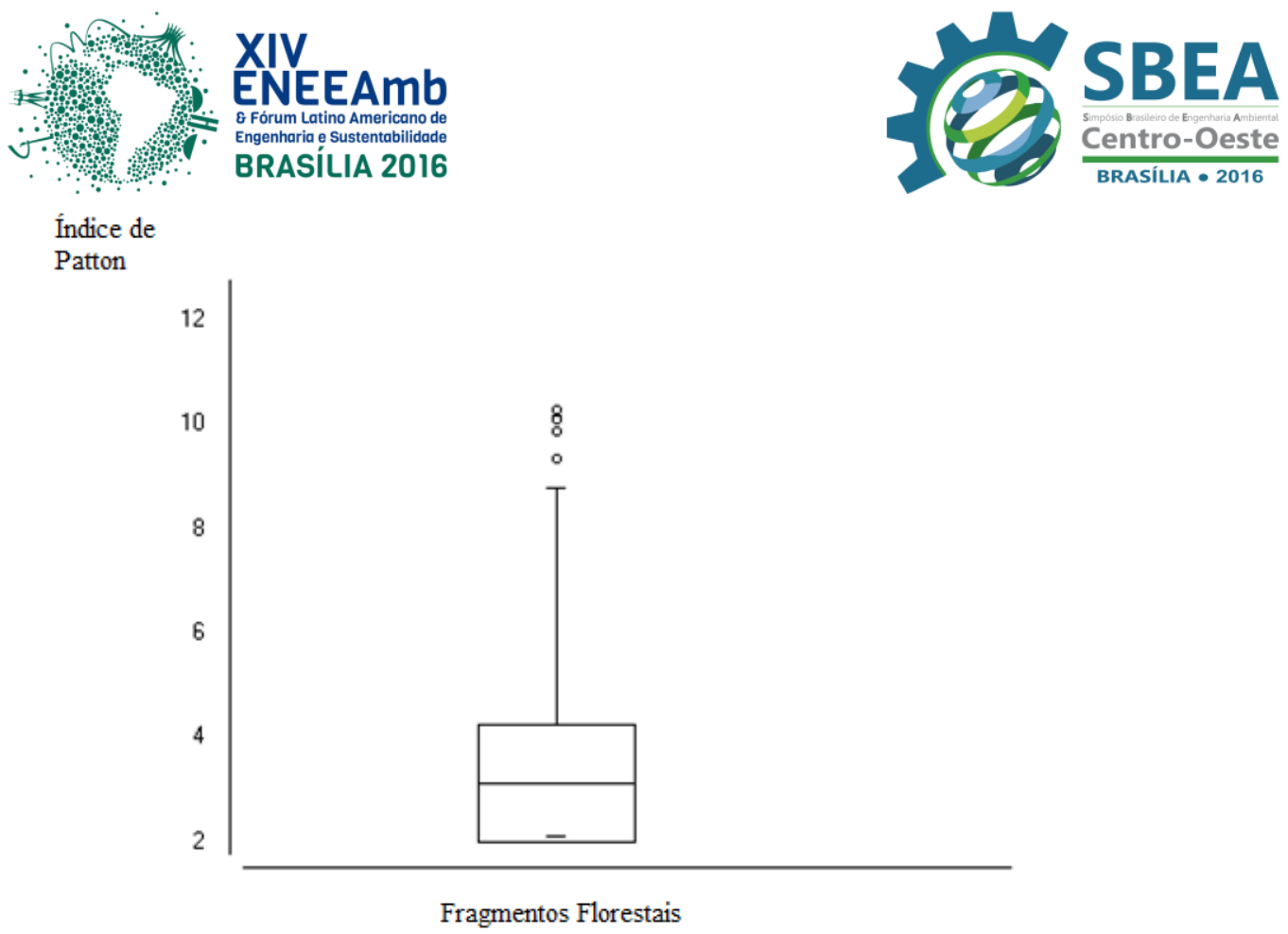

Figura 4 - Índice de Patton dos fragmentos florestais da Bacia do Rio Claro, PR.

Quanto mais próximo de um o índice de Patton, maior a tendência circular do fragmento. Assim, o maior valor obtido representa a área mais complexa, ou seja, a parcela mais negativamente afetada por sua forma. Para os fragmentos com maior grau de complexidade de forma são esperadas influências diretas geradas pelo efeito de borda, o que pode impactar na dinâmica da biodiversidade local por oferecer melhores condições para o estabelecimento de espécies especialistas de borda (LAURENCE et al., 2002; LAURENCE et al., 2007; RICKLEFS, 2006).

Com a métrica densidade da mancha obteve-se o valor de 2,06×10-7. A densidade é computada dividindo o número de manchas pelo total da área. A partir dessa métrica, observa-se que a sub-bacia em estudo apresenta fragmentos dispersos e desconectados, tendo em vista que a área total é extremamente maior que a soma das extensões dos fragmentos. Essa desconexão desfavorece o contato entre espécies da fauna, o que pode justificar o valor obtido para a métrica do tamanho efetivo da malha $\left(0,89 \mathrm{~km}^{2}\right)$ já que, de acordo com Jaeger (2000), tal métrica é baseada na habilidade de dois animais que habitam áreas diferentes em uma região se encontrarem dentro da paisagem. Consequentemente, a dispersão de sementes e a troca gênica dos vegetais se reduzem, resultando na perda da biodiversidade.

\section{CONSIDERAÇÕES FINAIS}

A sub-bacia do rio Claro é, em sua totalidade, intensamente fragmentada, com manchas florestais estreitas, que sofrem maior efeito de borda. Foi observado grande distância entre os remanescentes florestais, em geral, com a ausência de corredores ecológicos. Esse déficit de corredores dificulta o contato entre espécies de diferentes áreas, e até mesmo o isolamento geográfico de tais. Como manobra dessa situação, sugere-se a implantação de corredores ecológicos para que seja facilitado o fluxo de animais e de dispersão de sementes. 


\section{REFERÊNCIAS E CITAÇÕES}

BATISTA, M. Modelação Geográfica em Processos de Caracterização e Avaliação da Paisagem numa Perspectiva Transfronteiriça. Tese apresentada à Universidade de Évora. Évora, 2014.

FERREIRA, I. J. M.; COUTO, E. V.; VIEIRA-COUTO, L. M. Landscape Ecology Analysis of Mata Atlantica's Patches From South Brazil Using Free GIS. In: LATIN AMERICAN REMOTE SENSING WEEK - LARS, 3., 2016, Santiago, Chile. Anais... .Santiago, Chile. 2016. p. 1 - 5.

Instituto Agronômico do Paraná - IAPAR. Classificação Climática. Disponível em: http://www.iapar.br/modules/conteudo/conteudo.php?conteudo=863. Acesso em: 10 nov. 2015.

INSTITUTO BRASILEIRO DE GEOGRAFIA E ESTATÍSTICA. Manual Técnico da Vegetação Brasileira. Manuais Técnicos em Geociências. Rio de Janeiro, n. 1, ed. 2, 2012.

Instituto de Terras Cartografia e Geociências - ITCG. Solos - Estados do Paraná. Disponível em: http://www.iapar.br/modules/conteudo/conteudo.php?conteudo=863. Acesso em: 10 nov. 2015.

IRGANG, G.V.; MICOL, L.; SANTOS, R.R.dos. Análise da fragmentação da paisagem e mapeamento do valor para a conservação. Cuiabá: Icv, 2007. 24 p.

JAEGER, J. A. G. Landscape division, spliting index, and effective mesh size: new measures of landscape fragmentation. Landscape Ecology, n. 15, p. 115 - 130. Netherlands, 2000.

LAURANCE, W. F.; LOVEJOY, T. E.; VASCONCELOS, H. L.; BRUNA, E. M.; DIDHAM, R. K.; STOUFFER, P. C.; GASCON, C.; BIERREGAARD, R. O.; LAURANCE, S. G; SAMPAIO, E. Ecosystem Decay of Amazonian Forest Fragments: a 22-Year Investigation. Conservation Biology, 16: 605-618. 2002. doi: 10.1046/j.15231739.2002.01025.x

LELI, I. T.; STEVAUX, J. C.; DA NÓBREGA, M. T. Dinâmica espacial da hidrologia da bacia do Rio Ivaí. Boletim de Geografia, v. 28, n. 2, p. 41-47, 2010.

PETERKA, C. R. L. Avaliação do efeito da fragmentação florestal na diversidade de carrapatos e patógenos transmitidos por carrapatos na região do Pontal do Paranapanema, SP. 2008. 43 f. dissertação (Mestrado em Medicina Veterinária) - Faculdade de Medicina Veterinária e Zootecnia, Universidade de São Paulo, São Paulo, 2008.

RICKLEFS, R.E. A Economia da Natureza. Guanabara Koogan, ed. 6. Rio de Janeiro, 2006. 